International Journal of Medical Sciences

ISSN 1449-1907 www.medsci.org 2006 3(2):63-68

Review

(C)2006 Ivyspring International Publisher. All rights reserved

\title{
A Practical Approach to Managing Patients with HCV Infection
}

\author{
Richard H. Huang, and Ke-Qin Hu \\ Division of Gastroenterology and Hepatology, University of California, Irvine Medical Center, CA 92868, USA
}

Corresponding address: Ke-Qin Hu, MD, Director of Hepatology Services and Associate Professor of Clinical Medicine, Division of Gastroenterology, Univ. of California, Irvine Medical Center, 101 The City Drive, Building 53, Suite 113, Orange, CA 92868, USA. Phone: 714-456-6745. Email: kqhu@uci.edu

Received: 2005.12.30; Accepted: 2006.03.01; Published: 2006.04.01

Hepatitis C virus (HCV) infection is a major worldwide public health concern. It is a common cause of chronic liver disease and hepatocellular carcinoma. HCV antibody and HCV RNA testing are available diagnostic studies that offer high degree of accuracy. Current standard therapy includes a combination of pegylated interferon and ribavirin. Response rate is approximately $40 \%$ for genotype 1 and $80 \%$ for genotypes 2 and 3, respectively. Successful treatment can stop the progression of chronic liver disease, reduce the need for liver transplantation, and possibly decrease the risk for Hepatocellular carcinoma (HCC). Evaluating for potential treatment candidacy is an important initial step in the management of chronic HCV infection as not all individuals may need or qualify for the treatment. Understanding the natural history, the different diagnostic modalities, the current therapeutic options and, the treatment response and adverse effect profiles can help the practitioners better manage chronic HCV infection.

Key words: Chronic Hepatitis C, treatment, interferon, PEG-interferon, ribavirin

\section{Introduction}

Hepatitis $\mathrm{C}$ virus (HCV) infection, formerly known as non- $\mathrm{A}$, non-B hepatitis, was first identified in 1988. The WHO estimates that over 170 million people globally are chronically infected with $\mathrm{HCV}$, with 3-4 million new cases arising each year. Infection with $\mathrm{HCV}$ is a major cause of chronic liver disease that is associated with cirrhosis and hepatocellular carcinoma (HCC). While much effort has focused on the development of therapeutic agents, eradication of HCV infection remains a challenge. This article outlines in a systematic manner the clinical approach in the management of chronic HCV infection with review of the current practice guidelines, the available therapeutic options, and the development of new therapeutic strategies.

\section{Natural History of HCV Infection}

The natural history of HV infection was thoroughly reviewed by Drs. Chen and Morgan in this issue. Knowing the natural history of $\mathrm{HCV}$ infection is important in the management of patients with chronic $\mathrm{HCV}$ infection. Unfortunately, the natural history of chronic HCV infection is incompletely defined owing to the lack of long term prospective studies. Retrospective studies have provided a wide range estimate of the proportion (17 to 55\%) of chronic infection leading to cirrhosis within 20 years. The proportion estimated by the available prospective studies is much less. The NIH Consensus Statement in 2002 estimates the rate of development of cirrhosis in the order of 10 to $15 \%$.

Current natural history data suggest that progression of HCV infection is slow; the time course for the development of cirrhosis is estimated to be in the order of 20 to 30 years. Although the mortality related to liver disease in the infected population is described as higher (4.1 versus 1.3 percent) [1] than age-matched control population, the overall mortality has shown to be similar [2]. This may suggest that not all patients infected with
$\mathrm{HCV}$ develop chronic infection or have the same degree of liver disease progression.

Several factors have been identified to influence the rate of progression to cirrhosis in the $\mathrm{HCV}$ chronically infected population. Unfavourable factors include male age, age $>40$ years at infection, significant alcohol consumption $>30 \mathrm{~g} /$ day, and co-infection with human immunodeficiency virus (HIV) or hepatitis B virus (HBV) [3-6]. Recent data also identified diabetes and obesity as unfavourable risk factors [7]. On the other hand, female sex and younger age at infection are regarded to be associated with a lower rate of progression to cirrhosis [8]. No association has been shown between the progression of liver disease and HCV RNA and ALT levels.

Those with chronic HCV infection that progress to cirrhosis, most but not all, develop subsequent complications that eventually lead to hepatic decompensation. The most common indication of decompensation is usually ascites. Other complications that follow include variceal bleeding, encephalopathy and jaundice [9]. With the onset of cirrhosis, HCC occurs at a rate of $1-4 \%$ per year [10].

\section{Clinical Presentation of HCV Infection}

HCV infection may result in an acute episode of hepatitis which usually runs a mild clinical course with minimal risk of developing fulminant hepatic failure. However, the risk of developing a chronic infection after an acute episode of hepatitis $\mathrm{C}$ is high. Positive HCV RNA has been shown in 80 to 100 percent of those infected, and up to 80 percent with persistently elevated serum aminotransferase values [11,12].

Most patients with chronic $\mathrm{HCV}$ infection experience no symptoms. The remaining has mild and non-specific symptoms such as fatigue, arthralgia, myalgia, anorexia weight loss and depression. It is unclear whether these symptoms can be solely attributed to HCV infection or whether they are caused by a concomitant medical or psychiatric condition. However, these symptoms are 
reported to affect the quality of life which in turn can be improved after treatment $[13,14]$.

Symptoms in HCV infection do not appear to be linked to disease activity; neither do appear to be correlated with serum aminotransferase levels or liver histological findings $[15,16]$. In addition, serum aminotransferase level has not been shown to predict liver histological status $[17,18]$. The proportion of serum aminotransferase level elevation varies among patients with chronic HCV infection. Approximately one third of those chronically infected never develop elevated aminotransferase levels, while the remaining has a wide range of levels. Histological evidence of chronic inflammation is present in all patients but typically to a lesser degree in the group with normal serum aminotransferase levels [16].

Once cirrhosis develops, symptoms may be more evident and hepatic decompensation develops at a rate of approximately 4 percent per year but not in all patients [9]. The first sign of decompensation is usually ascites, subsequently followed by upper gastrointestinal bleeding, encephalopathy and jaundice. Hepatic decompensation leads to increased mortality in the chronic $\mathrm{HCV}$ infected population while HCC, which rarely occurs in the noncirrhotic, accounts for the increased mortality as well.

\section{Diagnosis of HCV Infection}

\section{Screening}

Routine screening should be directed to a population with a higher prevalence of $\mathrm{HCV}$ infection than the general population [19]. There are several high-risk groups for HCV infection. Individuals with a history of transfusion of clotting factors prior to 1987, as well as blood products or received organ transplantation prior to 1992 should be tested. Patients with a recent or remote history of injection drug use, on chronic hemodialysis, infants born to mothers with HCV or those with evidence of unexplained liver disease should all be screened as well. In addition, all patients with HIV infection should be screened since approximately $5-10 \%$ of those with positive HCV infection are co-infected with HIV [20].

\section{Testing Strategies}

\section{1) Serologic Testing}

The first-line diagnostic approach in the clinical setting is the utilization of the enzyme-linked immunoassay (ELISA) test, which tests for antibodies to $\mathrm{HCV}$. The current third generation of ELISA provides a sensitivity and specificity of $99 \%$ in as early as 7 to 8 weeks post infection [21].

\section{2) Molecular Testing}

Those who are positive for anti-HCV can then have HCV RNA levels tested to document viremia. Detection of HCV RNA levels can be accomplished by reverse transcription polymerase chain reaction (PCR) tests, which are highly sensitive. These tests are not only useful in the confirmation of $\mathrm{HCV}$ infection, but also in the evaluation of response to antiviral therapy when patients are undergoing treatment. In addition, there are instances when immuno-competency is in question, a negative anti$\mathrm{HCV}$ can be further evaluated by a HCV RNA test. HCV RNA can be measured either quantitatively or qualitatively, which can approach a low limit of detection of $50 \mathrm{IU} / \mathrm{ml}$ or 100 viral copies/ml as early as $1-2$ weeks post infection [21]. Therefore, when acute infection is suspected, HCV RNA can be tested before the appearance of HCV antibodies.

\section{3) Genotype Testing}

Once HCV infection is confirmed, the genotype of the HCV virus could be determined. The genotype of the $\mathrm{HCV}$ virus could play an important role when planning for treatment since it can predict the response rate to treatment and can guide the length of treatment [22-26]. There are six major genotypes of $\mathrm{HCV}$ currently recognized, numbered 1-6, and many subtypes. The prevalence of a particular genotype varies geographically. In the United States and Europe, over $90 \%$ of the HCV infections are of genotypes 1, 2 and 3, with genotype 1 being the most common. Genotype 4 is common in Africa and the Middle East, while genotypes 5 and 6 are the least prevalent and are found primarily in South Africa and Southeast Asia respectively.

\section{4) Role of Liver Biopsy}

A liver biopsy is not required for the diagnosis of $\mathrm{HCV}$ infection. Although several histopathological features of $\mathrm{HCV}$ infection have been described, these are non-specific and cannot be relied on in making a diagnosis [27]. However, a liver biopsy is routinely done prior to the treatment of $\mathrm{HCV}$ infection, although the utility of it is still debated. Regardless, a liver biopsy can provide several benefits. The histological findings can determine the staging of the liver disease and hence can provide the prognosis. If cirrhosis is found in a liver biopsy, patients can be screened periodically for hepatocellular carcinoma, since most will happen after the onset of cirrhosis. Periodic screening of esophageal varices is also warranted with the findings of cirrhosis. In addition, the liver biopsy can help in the selection of patients for treatment. Those patients found to have mild chronic hepatitis on liver biopsy can often opt to be monitored rather than treated, particularly if they have other comorbidities. Furthermore, a pre-treatment liver biopsy can help guide the treatment process particularly when patients are experiencing adverse effects. For example, in patients with advanced stage of liver disease, the treatment should be planned timely and the threshold to discontinue treatment should be higher since effective treatment will slow down disease progression. Liver biopsy may also help in diagnosing other co-existing liver diseases, like hepatic steatosis, a common presentation in $\mathrm{HCV}$ infected patients.

\section{Approach to Treatment of Chronic HCV Infection Initial Evaluation of Patients with Chronic HCV Infection}

1) History

Obtaining a thorough clinical history is important as the initial step in consideration for treatment. The history can provide clues regarding the timing of $\mathrm{HCV}$ infection and hence the possible duration of chronic liver disease which can give a more accurate prognosis. Elements in the history that help identify the possible timing of the infection or the duration of the liver disease include: prior history of injection drug use, former history of transfusion of blood products, unexplained history of abnormal liver transaminases, or a known history of contact exposure to $\mathrm{HCV}$. The clinical history can also disclose other concurrent medical conditions that may pose as barriers for treatment such as major depressive disorders, 
underlying autoimmune diseases, thyroid disorders, pregnancy, etc. In addition, the history can identify other factors such as alcohol use or abuse which may suggest possible accelerated disease progression or hints decreased responsiveness to treatment.

\section{2) Physical Examination}

The physical exam can further add information regarding the severity of the liver disease; unfortunately, physical signs may not be evident until patients develop cirrhosis. The presence of ascites and encephalopathy are diagnostic of decompensated liver disease. Other physical signs that may suggest cirrhosis and portal hypertension include the presence of splenomegaly, firm liver edge and spider angioma.

\section{3) Laboratory Tests}

Laboratory studies can provide useful values to predict the progression of liver disease. Laboratory studies should include a complete blood count (CBC), prothrombin time $(\mathrm{PT})$, international normalized ratio (INR), and a liver panel. Although the serum aminotransferase level correlates poorly with liver histology, the ratio of aspartate aminotransferase (AST) to alanine aminotransferase (ALT) $>1$ is a dependable marker for cirrhosis [28,29]. Increased INR and thrombocytopenia is also seen more frequently in cirrhosis [30]. Additional laboratory studies that are useful include a-fetoprotein (AFP) level, HCV genotype and RNA level. The AFP is widely used in screening of HCC, while HCV genotype and RNA level can later guide the treatment process.

\section{4) Radiographic Studies}

Several modalities of radiographic tests are used to evaluate patients with chronic HCV. Ultrasound is useful in the evaluation for evidence of portal hypertension such as splenomegaly, recanalization of the umbilical vein, ascites, etc. It is also useful in the screening of HCC although CT scan or MRI provides higher sensitivity for this purpose [31]. Liver-Spleen scans can provide additional information when cirrhosis is suspected. Colloid shifting and evidence of splenomegaly is suggestive of cirrhosis.

\section{Selection of Patients for Treatment of Chronic HCV}

\section{1) Why to Treat HCV Infection}

The treatment of $\mathrm{HCV}$ is justified by the natural history and the outcome of chronic infection and liver injury that is associated with cirrhosis, hepatic decompensation, development of HCC, and mortality. In addition, HCV infection has been linked to a variety of extra-hepatic manifestations such as autoimmune diseases, lymphoma, monoclonal gammopathies and cryoglobulinemia. Some of these extra-hepatic complications could lead to severe systemic disease and damage to other organs before significant liver disease is evident. These extra-hepatic manifestations could also impair significantly the quality of life. The goal of treatment is to stop or slow down disease progression and prevent complications due to chronic $\mathrm{HCV}$ infection by sustained suppression of HCV replication.

\section{2) Who Should Receive Treatment}

As a general rule, treatment should be considered for all patients with virologic or histologic evidence of chronic infection. Therefore, every patient with chronic HCV is a potential candidate for treatment. The decision to treat is never clear-cut, but rather it is weighed by the severity of disease, the predicted success rate, the anticipated adverse effects and the cost and availability of the drugs. The decision to treat should never be made by the physician alone; it should be made only after educating patients regarding the disease, the prognosis, the treatment options, the outcomes and the alternatives if chose not to be treated (Fig 1). Taking all these factors in consideration, there are several guidelines currently available to help decide which population of patients should undergo therapy. The American Association for the Study of Liver Diseases (AASLD) recognizes three categories of patients: those in whom therapy is widely accepted, those in whom therapy should be individualized and, those in whom therapy is contraindicated [32].

Based on these guidelines, therapy is widely accepted for those who are at least 18 years old, who have evidence of abnormal ALT values, whose liver biopsy shows chronic hepatitis with significant fibrosis (Metavir score $\geq 2$, Ishak score $\geq 3$ ), who have compensated liver disease (total serum bilirubin $<1.5 \mathrm{~g} / \mathrm{dL}$, INR $<1.5$, albumin $>3.4 \mathrm{~g} / \mathrm{dL}$, platelet count $>75,000 \mathrm{k} / \mathrm{mm}^{3}$, and no evidence of hepatic encephalopathy or ascites). In addition, patients should have acceptable hematological and biochemical indices (hemoglobin $>13 \mathrm{~g} / \mathrm{dL}$ for men and $>12 \mathrm{~g} / \mathrm{dL}$ for women, neutrophil count $>1.5 \mathrm{k} / \mathrm{mm} 3$, creatinine $<1.5 \mathrm{mg} / \mathrm{dL}$ ). If there is a pre-existing history of depression, it should be well controlled. When patients have been treated previously for HCV infection, they should be considered as long as all the above criteria are met. However, the most important factor for consideration of treatment is the patient's willingness to be treated and to conform to the treatment requirements.

Treatment is generally contraindicated in patients with major uncontrolled depressive disorders, untreated hyperthyroidism or any other severe concurrent disease, such as poorly controlled hypertension or diabetes, obstructive pulmonary disease, or significant heart disease. Patients with autoimmune hepatitis or other conditions known to be exacerbated by interferon and ribavirin or known to have hypersensitivity to these drugs should not receive treatment. Treatment is also contraindicated in those who are post renal, heart, or lung transplant recipients. In addition, children under 3 years of age, pregnant patients or those unwilling to comply with adequate contraception should not be treated either.

Treatment should be individualized in patients with the following characteristics: 1) acute HCV infection; 2) coinfection with HIV; 3 ) under 18 years of age; 4 ) chronic renal disease, on or not on hemodialysis; 4) decompensated cirrhosis; 5) liver transplant recipients; 6) persistently normal ALT values; 7) failed prior treatment and; 8) current users of illicit drugs or alcohol but willing to participate in substance abuse programs. Some of these were further discussed by Dr. Hoefs et al in the separated article in this issue.

\section{3) Available Antiviral Therapy}

The current standard of therapy includes the combination of weekly pegylated interferon and daily ribavirin. The treatment duration and dosage, as well as the response rate depend on $\mathrm{HCV}$ genotype, $\mathrm{HCV}$ load and other factors. The pegylated interferon is an interferon that has a polyethylene glycol moiety attached, 
this results in a longer half-life and improved pharmacokinetics which allows once-weekly subcutaneous injections as compared to the non-pegylated interferons, which required three times weekly injections.

There are currently two available pegylated interferons, $a 2 a$ and $a 2 b$. Two large randomized studies compared interferon plus ribavirin with pegylated interferon plus ribavirin $[24,25]$. One study tested a2a (fixed dose of $180 \mu \mathrm{g}$ ) with ribavirin dose adjusted based on weight (1000 mg for weight $<75 \mathrm{~kg}, 1200 \mathrm{mg}$ for weight $>75 \mathrm{~kg}$ ); while the other tested $\mathrm{a} 2 \mathrm{~b}$ (adjusted by weight at $1.5 \mu \mathrm{g} / \mathrm{kg}$ ) with a fixed dose of ribavirin of $800 \mathrm{mg}$. Both studies reported similar efficacies as well as adverse effect profiles. The sustained virological response (SVR) rates with the combination of pegylated interferons and ribavirin are reported to be much superior as compared with the combination of interferon and ribavirin. The SVR were approximately $40 \%$ for genotype 1 and $80 \%$ for genotypes 2 and 3, respectively [24,25]. However, there are no reported studies with direct comparisons between these two pegylated interferons. The variables reported to be associated with SVR include a genotype other than 1 , viral load $<2$ million copies/ml, age $<40$, and lower body weight. In addition, these studies provided evidence that early virological response (EVR) defined as $\mathrm{HCV}$ viral load is decreased by at least 2 log or to an undetectable level at 12 weeks of treatment predicts a high possibility of SVR.

In a separate study, pegylated interferon a2a was used with ribavirin either adjusted by weight or fixed dose of $800 \mathrm{mg}$, for 24 vs. 48 weeks [26]. From this study, it appears that SVR rate for genotype 1 was significantly superior when a2a peg-interferon was given with weightadjusted dose of ribavirin for 48 weeks vs. when given for 24 weeks or when given with low dose of $800 \mathrm{mg}$ of ribavirin. However, for genotypes 2 and 3, SVR rate was similar regardless of the dose of ribavirin or the duration of the treatment. Besides genotypes 1, 2 and 3, other genotypes were not representative in the above mentioned studies, but in general, genotypes 4,5 and 6 are thought to respond more like genotype 1 . Thus, 48weeks treatment is recommended for these genotypes.

The current guidelines support the use of a pegylated interferon a plus ribavirin as the first line therapy. For genotype 1, ribavirin should be dosed based on weight (i.e., $1000 \mathrm{mg} /$ day for weight $<75 \mathrm{~kg}, 1200 \mathrm{mg}$ for weight $>75 \mathrm{~kg} /$ day), and the duration of treatment should be anticipated for 48 weeks. On the other hand, genotypes 2 and 3 will require only ribavirin $800 \mathrm{mg} /$ day, and the duration of treatment is recommended for 24 weeks [32].

\section{4) Follow-up of Treatment}

Once treatment has been decided for a particular patient, routine follow-up is planned. Pre-treatment baseline laboratory values should be obtained including CBC, complete metabolic panel, PT and HCV viral load. Patients should return 2 weeks after initiation of therapy and subsequently every 4 weeks. At these encounters, the above routine laboratory studies should be obtained as well to monitor for signs of adverse effects due to the drugs. Repeat HVC viral load should be repeated at week 12 of treatment to document EVR. The decision to continue treatment after week 12 would then be determined based on individual response to treatment (EVR), tolerance to the drugs, laboratory profiles, and the pre-treatment assessment of the severity of the liver disease. During each follow-up, signs and symptoms of possible adverse effects should be evaluated. If treatment is continued in the presence of adverse effects, dose adjustments can be considered. On the other hand, a particular adverse effect can be treated or monitored without lowering medication dosages depending on its severity. Once the course of treatment is completed, a qualitative $\mathrm{HCV}$ viral load must be obtained to document end of treatment response (ETR). The same test should be done 6 months later to evaluate for SVR.

\section{5) General Measures}

Several general measures should be addressed for every patient with chronic $\mathrm{HCV}$ infection regardless of treatment candidacy. Alcohol abstinence should be recommended to all patients as this may accelerate the progression of liver disease. Hepatotoxic drugs should also be avoided, as patients with advanced fibrosis have little reserve for additional injury. Immunization against hepatitis A and B should be given if patients do not exhibit markers of prior exposure. Although there is no data to support a particular diet for patients with HCV infection, those who have late stage liver disease may be counselled regarding low sodium diet to avoid fluid retention. In general, a healthy diet is recommended particularly for those with high body mass index since there is data suggesting that obesity accelerating fibrosis.

\section{Research Direction}

Although significant advances in therapeutic options have improved the outcome in the management of HCV infection, many challenges remain ahead. The proportion of chronic HCV not responding to the available drugs is far from insignificant, particularly for genotypes other than 2 and 3. Furthermore, these drugs are not devoid of adverse effects, some of which are severe enough to warrant discontinuation of the treatment. In addition, the large proportion of patients with comorbidities and those who fall into the category of "special groups", represent even greater challenge. On the other hand, it is encouraging that new therapeutic options continue to emerge. Viramidine is a prodrug of ribavirin that does not accumulate in erythrocytes and is preferentially converted to ribavirin in liver. A phase II study indicated a lower rate of anemia induced by viramidine than ribavirin. However, its efficacy remains to be determined by ongoing clinical trials. Several types of HCV-specific protease inhibitors have been developed. Although the early results appear very promising, their safety and efficacy remain to be confirmed by phase III trials. In addition, active research is being focused on searching new anti-HCV agents. The ideal therapy in the future will likely focus on drugs with high response rate for both treatment naïve and treatment refractory patients, while providing an improved adverse effect profile and a shorter duration of treatment.

\section{Conflict of interest}

The authors have declared that no conflict of interest exists.

\section{References}

1. Seeff LB, Hollinger FB, Alter HJ, et al. Long-term mortality and morbidity of transfusion-associated non-A, non-B, and type $C$ hepatitis: A National Heart, Lung, and Blood Institute collaborative study. Hepatology 2001;33(2):455-63. 
2. Seeff LB, Miller RN, Rabkin CS, et al. 45-year follow-up of hepatitis C virus infection in healthy young adults. Ann Intern Med 2000;132(2):105-11.

3. Poynard T, Bedossa P, Opolon P. Natural history of liver fibrosis progression in patients with chronic hepatitis C. The OBSVIRC, METAVIR, CLINIVIR, and DOSVIRC groups. Lancet 1997;349(9055):825-32.

4. Benhamou Y, Bochet M, Di Martino V, et al. Liver fibrosis progression in human immunodeficiency virus and hepatitis $C$ virus coinfected patients. The Multivirc Group. Hepatology 1999;30(4):1054-8.

5. Zarski JP, Bohn B, Bastie A, et al. Characteristics of patients with dual infection by hepatitis B and C viruses. J Hepatol 1998;28(1):2733.

6. Wiley TE, McCarthy M, Breidi L, et al. Impact of alcohol on the histological and clinical progression of hepatitis $\mathrm{C}$ infection. Hepatology 1998;28(3):805-9.

7. Bellentani S, Pozzato G, Saccoccio G, et al. Clinical course and risk factors of hepatitis $C$ virus related liver disease in the general population: report from the Dionysos study. Gut 1999;44(6):874-80.

8. Kenny-Walsh E. Clinical outcomes after hepatitis $C$ infection from contaminated anti-D immune globulin. Irish Hepatology Research Group. N Engl J Med 1999;340(16):1228-33.

9. Fattovich G, Giustina G, Degos F, et al. Morbidity and mortality in compensated cirrhosis type C: a retrospective follow-up study of 384 patients. Gastroenterology 1997;112(2):463-72.

10. Lauer GM, Walker BD. Hepatitis C virus infection. N Engl J Med 2001;345(1):41-52.

11. Farci $\mathrm{P}$, Alter HJ, Wong D, et al. A long-term study of hepatitis $C$ virus replication in non-A, non-B hepatitis. $N$ Engl J Med 1991;325(2):98-104.

12. Barrera JM, Bruguera M, Ercilla MG, et al. Persistent hepatitis C viremia after acute self-limiting posttransfusion hepatitis $C$. Hepatology 1995;21(3):639-44.

13. Spiegel BM, Younossi ZM, Hays RD, et al. Impact of hepatitis C on health related quality of life: a systematic review and quantitative assessment. Hepatology 2005;41(4):790-800.

14. Ware JE Jr., Bayliss MS, Mannocchia M, et al. Health-related quality of life in chronic hepatitis C: impact of disease and treatment response. The Interventional Therapy Group. Hepatology 1999;30(2):550-5.

15. Merican I, Sherlock S, McIntyre N, et al. Clinical, biochemical and histological features in 102 patients with chronic hepatitis $C$ virus infection. Q J Med 1993;86(2):119-25.

16. Shakil AO, Conry-Cantilena C, Alter HJ, et al. Volunteer blood donors with antibody to hepatitis $\mathrm{C}$ virus: clinical, biochemical, virologic, and histologic features. The Hepatitis C Study Group. Ann Intern Med 1995;123(5):330-7.

17. Haber $M M$, West $A B$, Haber $A D$, et al. Relationship of aminotransferases to liver histological status in chronic hepatitis C. Am J Gastroenterol 1995;90(8):1250-7.

18. McCormick SE, Goodman ZD, Maydonovitch CL, et al. Evaluation of liver histology, ALT elevation, and HCV RNA titer in patients with chronic hepatitis C. Am J Gastroenterol 1996;91(8):1516-22.

19. Recommendations for prevention and control of hepatitis $C$ virus (HCV) infection and HCV-related chronic disease. Centers for Disease Control and Prevention. MMWR Recomm Rep 1998;47(RR19):1-39.

20. Alter MJ, Kruszon-Moran D, Nainan OV, et al. The prevalence of hepatitis C virus infection in the United States, 1988 through 1994. N Engl J Med 1999;341(8):556-62.

21. Pawlotsky JM. Use and interpretation of virological tests for hepatitis C. Hepatology 2002;36(5 Suppl 1):S65-73.

22. McHutchison JG, Gordon SC, Schiff ER, et al. Interferon alfa- $2 b$ alone or in combination with ribavirin as initial treatment for chronic hepatitis C. Hepatitis Interventional Therapy Group. N Engl J Med 1998;339(21):1485-92.

23. Poynard T, Marcellin P, Lee SS, et al. Randomised trial of interferon alpha $2 \mathrm{~b}$ plus ribavirin for 48 weeks or for 24 weeks versus interferon alpha2b plus placebo for 48 weeks for treatment of chronic infection with hepatitis $C$ virus. International Hepatitis Interventional Therapy Group (IHIT). Lancet 1998;352(9138):1426-32.
24. Manns MP, McHutchison JG, Gordon SC, et al. Peginterferon alfa-2b plus ribavirin compared with interferon alfa- $2 b$ plus ribavirin for initial treatment of chronic hepatitis C: a randomised trial. Lancet 2001;358(9286):958-65.

25. Fried MW, Shiffman ML, Reddy KR, et al. Peginterferon alfa-2a plus ribavirin for chronic hepatitis $\mathrm{C}$ virus infection. N Engl J Med 2002;347(13):975-82.

26. Hadziyannis SJ, Sette H Jr., Morgan TR, et al. Peginterferon-alpha2a and ribavirin combination therapy in chronic hepatitis $\mathrm{C}$ : a randomized study of treatment duration and ribavirin dose. Ann Intern Med 2004;140(5):346-55.

27. Scheuer PJ, Ashrafzadeh P, Sherlock S, et al. The pathology of hepatitis C. Hepatology 1992;15(4):567-71.

28. Sheth SG, Flamm SL, Gordon FD, et al. AST/ALT ratio predicts cirrhosis in patients with chronic hepatitis C virus infection. Am J Gastroenterol 1998;93(1):44-8.

29. Giannini E, Risso D, Botta F, et al. Validity and clinical utility of the aspartate aminotransferase-alanine aminotransferase ratio in assessing disease severity and prognosis in patients with hepatitis $\mathrm{C}$ virus-related chronic liver disease. Arch Intern Med 2003;163(2):21824.

30. Adinolfi LE, Giordano MG, Andreana A, et al. Hepatic fibrosis plays a central role in the pathogenesis of thrombocytopenia in patients with chronic viral hepatitis. Br J Haematol 2001;113(3):590-5.

31. Harisinghani MG, Hahn PF. Computed tomography and magnetic resonance imaging evaluation of liver cancer. Gastroenterol Clin North Am 2002;31(3):759-76.

32. Strader DB, Wright $\mathrm{T}$, Thomas DL, et al. Diagnosis, management, and treatment of hepatitis C. Hepatology 2004;39(4):1147-71.

\section{Author biography}

Richard H. Huang, MD, is a fellow in the Department of Medicine, Division of Gastroenterology and Hepatology, University of California, Irvine, California, USA. His current research interests include the molecular pathways of chemoprevention of colorectal cancer.

Ke-Qin Hu, MD, is the Director of Hepatolgy Services and Associate Professor of Clinical Medicine, Division of Gastroenterology, University of California, Irvine, California, USA. His current research interests include the natural history and management of hepatitis $B$ and $C$ and chemoprevention of hepatocellular carcinoma. 


\section{Figure}

Figure 1. Algorithm for the management of chronic HCV infection

Evidence of abnormal serum aminotransferase

$+$

Risk factors for $\mathrm{HCV}$ infection

$\downarrow$

Test for HCV antibodies
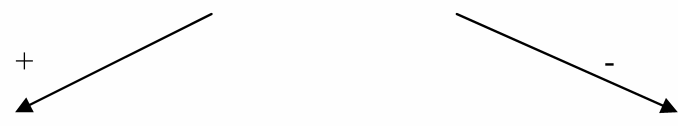

Confirm HCV PCR +

Confirm HCV PCR neg (if patient is not immuno-competent or has high risk factors and suspect false negative
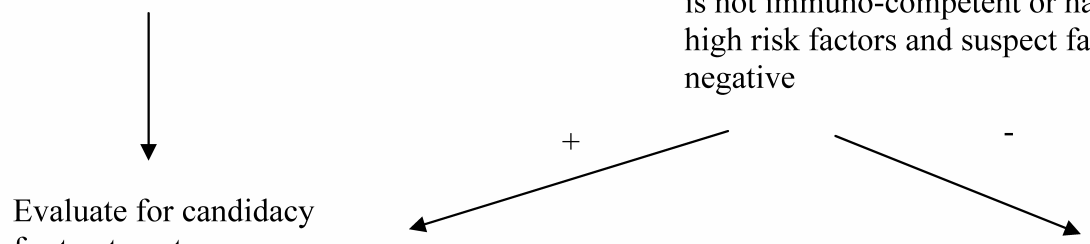
for treatment

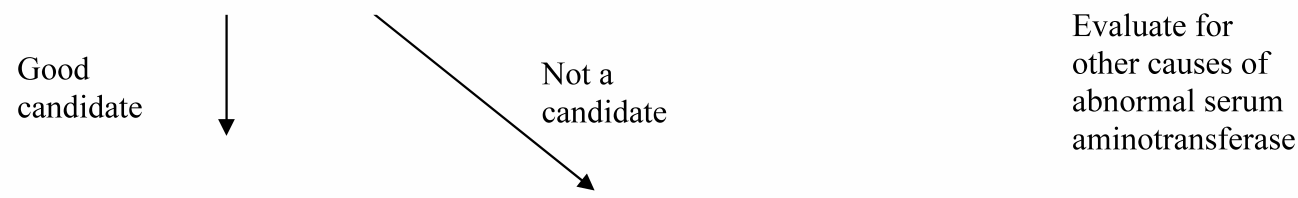

Test for genotype \pm liver biopsy

Liver biopsy with

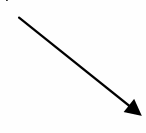

significant fibrosis

Start treatment after educating patient

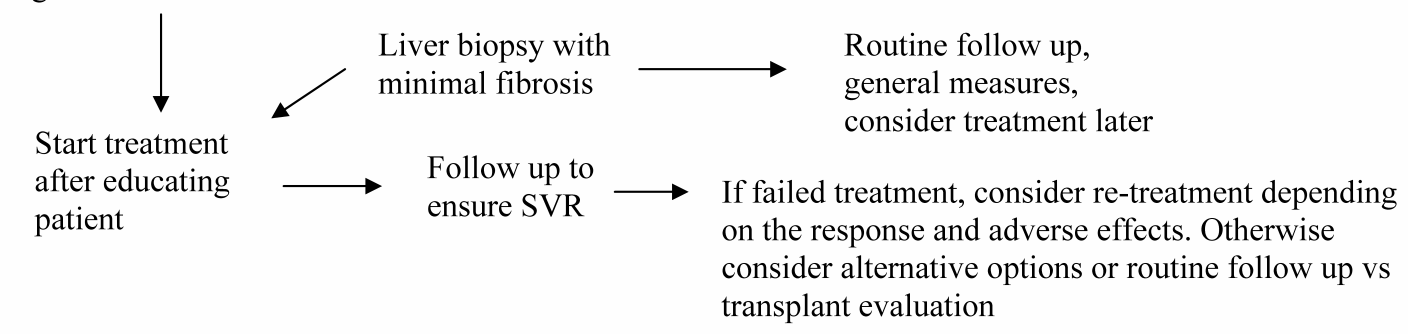

Medical management of symptoms, routine follow up for prevention of variceal bleeding, HCC. General measures. Transplant evaluation 